\title{
Bovine leukemia virus and cow longevity in Michigan dairy herds
}

\author{
P. C. Bartlett, ${ }^{, 1}$ B. Norby, ${ }^{*}$ T. M. Byrem,† A. Parmelee, ${ }^{*}$ J. T. Ledergerber, $†$ and R. J. Erskine ${ }^{*}$ \\ ${ }^{*}$ Department of Large Animal Clinical Sciences, Michigan State University, East Lansing 48824 \\ †Antel BioSystems Inc., Lansing, MI 48909
}

\section{ABSTRACT}

To determine the association between infection with bovine leukemia virus (BLV) and cow longevity, a stratified random sample of 3,849 Holsteins in 112 Michigan dairy herds was followed for an average of $597 \mathrm{~d}$ following testing for BLV antibodies with an ELISA milk test. The hazard ratio of 1.23 indicates that BLV-positive cows were $23 \%$ more likely than their BLV-negative herd mates to die or be culled during the monitoring period. This result is adjusted for lactation number, which is also positively associated with an increased risk of leaving the herd. Because herd was included in models, the effect of BLV ELISA on cow longevity was a within-herd comparison in which BLVinfected cattle were compared with their uninfected herd mates. The analysis of 4 ELISA optical density (OD) groups demonstrated a dose response such that cows with higher OD values had decreased survival compared with cows with lower OD values. Cows with OD values above 0.5 were at $40 \%$ greater risk of dying or being culled than were their uninfected herd mates. These results support the contention that the association of BLV with cow longevity, when added to other economic impacts, may warrant the control of BLV in our US dairy cow population.

Key words: leukosis, culling, survival analysis, welfare

\section{INTRODUCTION}

Enzootic bovine leukosis is a contagious disease of cattle caused by bovine leukemia virus (BLV), which is a retrovirus most often associated with subclinical infections in cattle. However, approximately 30 to $40 \%$ of BLV carriers will develop persistent lymphocytosis and less than 5\% develop malignant lymphosarcoma (Schwartz and Levy, 1994). Twelve countries in Europe have eradicated BLV through management changes and test-and-slaughter control programs (European Commission, 2003; Nuotio et al., 2003; Acaite et al., 2007).

Received July 11, 2012.

Accepted November 23, 2012

${ }^{1}$ Corresponding author: bartlett@cvm.msu.edu
Surveys from geographic locations other than Europe have reported within-herd cow prevalence of BLV in adult dairy cattle from 23 to $46 \%$ (Sargeant et al., 1997; Trono et al., 2001; Ott et al., 2003; VanLeeuwen et al., 2005). In Michigan, the cow prevalence of BLV was reported as 33\%. (Erskine et al., 2012a,c).

The total economic loss from BLV infection includes many cost components. Losses resulting from restrictions in trade or selection of animals used as genetic stock are difficult to quantify. Economic losses per case of lymphosarcoma were estimated to be $\$ 412$ (Rhodes et al., 2003). Losses associated with subclinical BLV infection are more difficult to quantify as they may be due to compromised immune function, resulting in an increased susceptibility to a wide variety of opportunistic pathogens. Losses associated with decreased milk production have been discussed in previous herdlevel analyses (Ott et al., 2003; Erskine et al., 2012a). However, cows with reduced milk production are usually culled before the full effect of their decreased milk production is realized. If one were to consider a partial budget for total BLV economic impact, the culling of poor milk-producing cows transfers the cost of poor production from the milk production component to the culling component. Likewise, reduced reproductive performance would mostly be realized as premature culling of cattle that do not become pregnant. Therefore, overall survival of BLV-infected dairy cattle may be a more comprehensive measure of the impact of BLV on a dairy herd in that it includes cow removal from the herd for a variety of reasons that may result from hypothesized BLV-altered immune function.

Previous studies determined that cull rates increased significantly as BLV-seropositive cows age (Thurmond et al., 1985) and that this may be especially important among BLV-infected cows that transform to lymphocytosis (Pollari et al., 1992). However, other studies have found that the association between BLV and the survival of dairy cows is not statistically significant (Tiwari et al., 2005). Our previous work (Erskine et al., 2012a) found a herd-level negative association between cow age (estimated as the percentage of the herd in the third-or-greater lactation) and BLV prevalence. These previous findings prompted the collection of cow-specif- 
ic data to determine if a cow-level association between BLV infection and cow longevity was evident.

\section{MATERIALS AND METHODS}

\section{Selection of Study Herds and Cows}

This study was part of a previously described database (Erskine et al., 2012a,c). In brief, dairy herds in Michigan that routinely participated in DHIA testing and averaged $\geq 120$ cows on test for the previous 12 mo were stratified into equal-sized cohorts of 119 small-sized herds (120-174 cows), 119 medium-sized herds (174-295 cows), and 119 large herds (298-6,738 cows). Within each of these strata, herds were assigned a random number, which determined the order in which they were contacted and invited to participate in our study. We sought approximately 40 herds from each cohort, although only 112 were available for study because of the inability to schedule herd visits, or lack of data within herd DHIA records for some of the selected herds. Within each herd, we identified up to 10 cows each from the first, second, third, and $\geq$ fourth lactations that were the most recently calved, based on the current DHIA test. On the next DHIA test day for each herd, (June through August, 2010) DHIA technicians collected milk samples from the selected cows for submission to the laboratory for ELISA testing of BLV antibodies (Erskine et al., 2012c). The ELISA optical density (OD) results were corrected by subtraction of the average of the negative controls, and values $>0.1$ were considered positive for previous BLV exposure. In the spring of 2012, DHIA records were accessed to determine the date of all culls and deaths. Herds were monitored for an average of $597 \mathrm{~d}$ (range 518 to 629). Excluded were 74 cows sold for use as dairy animals. Producers only received summary information on the BLV testing done for this study and were not told which animals were BLV positive. Only 26 of the producers reported a history of doing some BLV testing, and only 6 did so at least annually or at calving. Therefore, knowledge of the BLV status of individual cattle should not have had any sizable effect on culling decisions. It should be noted that our survival analysis began at the time of detection, not the unknown time of infection. Also, because of marketing-related regulations, dairy herds in the entire state of Michigan had been excluded from the use of bovine somatotropin (bST) throughout the 12-mo period before the initiation of our study in the summer of 2010 .

\section{Statistical Analyses}

The days to culling was analyzed using Stata software (version 11.0; StataCorp LP, College Station, TX) using nonparametric Kaplan-Meier survival graphs (sts graph procedure) and semi-parametric Cox proportional hazard models (stcox procedure) handling ties with the Breslow method. Kaplan-Meier graphs were used to visually assess the survival curves. Two Cox proportional hazard models, including a shared frailty (random effect) for herd, were used to analyze the effect of BLV status on survival in the herd. The first model used BLV ELISA (negative: OD $<0.1$; positive: $\geq 0.1$ ), and a second model used 4 BLV ELISA OD categories (OD $<0.1,0.1 \leq \mathrm{OD}<0.25,0.25 \leq \mathrm{OD}<$ 0.5 , and $\mathrm{OD} \geq 0.5)$. The main effects were estimated after controlling for lactation number $(1,2,3$, and $\geq 4)$. Additionally, the first-order interactions between BLV OD status and lactation were examined. Results were considered statistically significant at $P<0.05$.

The shared frailty model using multiplicative gammadistributed random effects on the hazard scale was used to model within-herd correlation among cows. Model diagnostics for the Cox models were based on Cox-Snell residuals, Schoenfeld residuals, as well as interaction terms between log-transformed time to event and all or each of the predictor variables (Cleves et al., 2010). Additionally, the same models were analyzed using SAS 9.3 PROC PHREP, with herd as a random effect variable and with PROC SURVEYPHREG with herd included as a cluster variable.

\section{RESULTS}

During the monitoring period, there were 3,849 cows with complete data, of which 1,860 died or were culled during the monitoring period, and 1989 were present when monitoring ceased for that particular herd. The 112 herds that were studied contributed an average of 35 cows to the study.

Of the BLV-negative cows, $44 \%(1,116 / 2,540)$ were culled, compared with $57 \%(744 / 1,309)$ of the BLVpositive cows. The analysis of BLV-negative versus BLV-positive cows is shown in Table 1 and Figure 1. The hazard ratio of 1.23 indicates that BLV-positive cows were $23 \%$ more likely than their BLV-negative herd mates to die or be culled during the average 597d monitoring period after being tested for BLV. This result is adjusted for lactation number, which is also positively associated with an increased risk of leaving the herd. Because herd was included in models, shared frailty, the effect of BLV ELISA on cow longevity, was a within-herd comparison in which BLV-infected cattle were compared with their uninfected herd mates. The analysis of the 4 OD groups is shown in Table 2 and Figure 2 and shows a dose response in that cows with higher OD values had worse survival than did cows with lower OD values. Cows with OD values above 0.5 


\section{Product-Limit Survival Estimates}

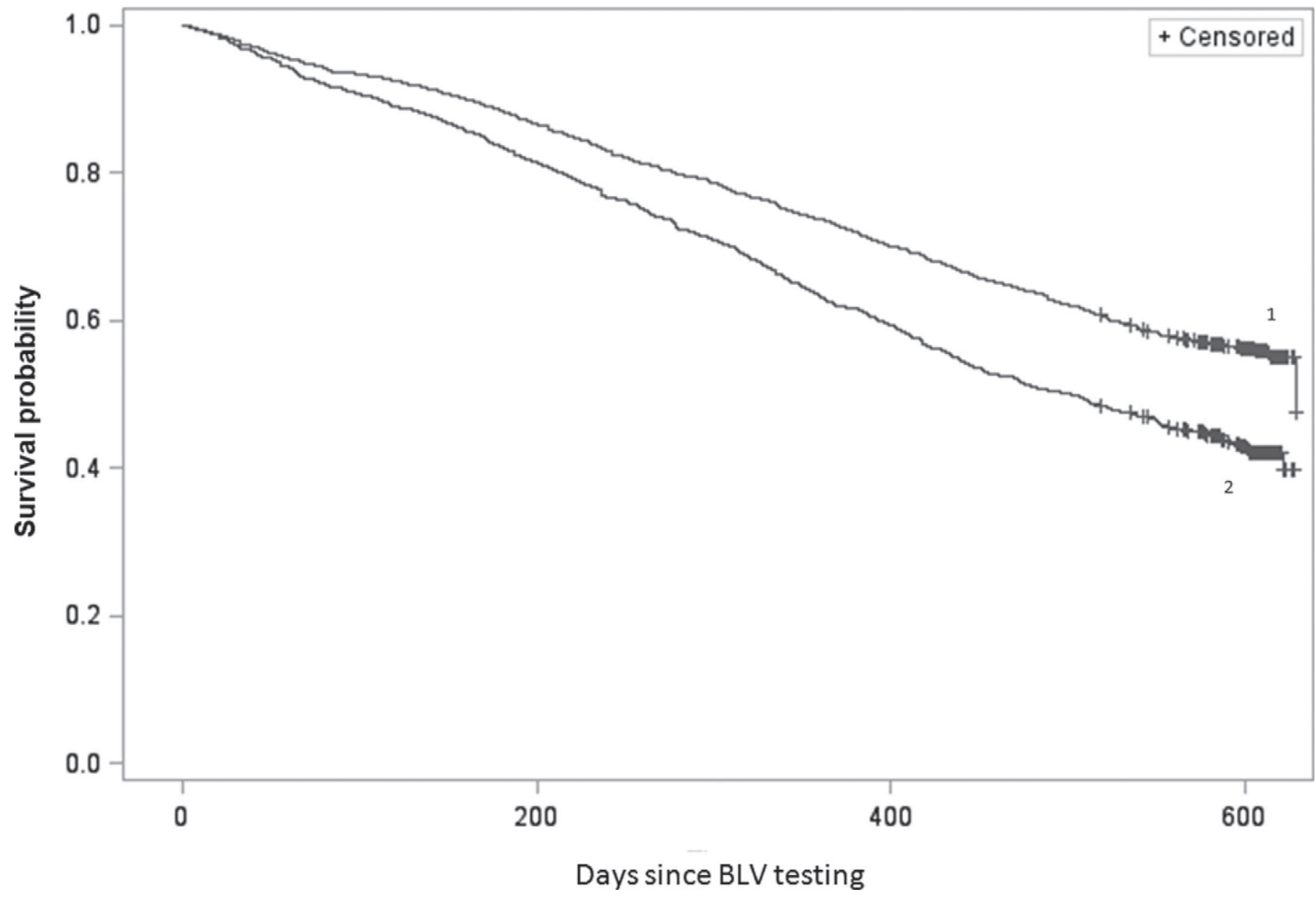

Figure 1. Survival of cattle following bovine leukemia virus (BLV) testing; proportion of cattle surviving (y-axis) and days since BLV testing (x-axis). ${ }^{1} \mathrm{BLV}$ negative (optical density $<0.1$ ); ${ }^{2} \mathrm{BLV}$ positive (optical density $\geq 0.1$ ).

were at $40 \%$ greater risk of dying or being culled than were their uninfected herd mates. None of the 2-way interactions between lactation groups and BLV (or OD groups) were significant at $P<0.1$.

Nonsignificant $P$-values $(P>0.05)$ for time-varying interactions between log-time, BLV status (or OD categories), and lactation number indicated that the basic assumption of proportional hazards were not violated. Visual assessment of the cumulative hazard function $\mathrm{H}(\mathrm{t})$ plotted against the Cox-Snell residuals indicated a good fit of the model.

The PHREG models produced hazard ratios that were identical, to 3 significant digits, as those for the Stata output shown in Tables 1 and 2. The SURVEYPHREG model agreed with the Stata model in finding a 1.23 hazard ratio for the model specified in Table 1; however, the hazard ratios for the Table 2 model were slightly different, by no more than 0.015 . The standard errors were smaller for both the PHREG and SURVEYPHREG models.

\section{DISCUSSION}

Some single-farm studies have found an association between BLV and cow longevity (Da et al., 1993; Pollari et al., 1993). However, it is unknown to what extent such studies may be representative of farms with different environmental and management conditions. Pollari et al. (1991) reported that earlier culling may be a result of persistent lymphocytosis. Huber et al. (1981) saw no evidence of an association between BLV and reduced cow longevity.

The study by Tiwari et al. (2005) showed a graphical trend toward reduced longevity among BLV-positive cows. This association remained, but was not statistically significant $(P<0.30)$, when included in a 
Table 1. Survival of cattle following bovine leukemia virus (BLV) testing (negative or positive) for milk ELISA antibodies

\begin{tabular}{|c|c|c|c|c|c|}
\hline Effect & $\begin{array}{c}\text { Hazard } \\
\text { ratio }\end{array}$ & $\mathrm{SE}$ & $\mathrm{Z}^{1}$ & $P$-value & $95 \%$ CI \\
\hline$\overline{\mathrm{OD}<0.1^{2}}$ & - & - & - & - & - \\
\hline $\mathrm{OD} \geq 0.1^{3}$ & 1.23 & 0.0633 & 3.93 & 0.000 & $1.11-1.36$ \\
\hline Lactation 1 & - & - & - & - & - \\
\hline Lactation 2 & 1.72 & 0.135 & 7.04 & 0.000 & $1.48-2.01$ \\
\hline Lactation 3 & 2.55 & 0.193 & 12.35 & 0.000 & $2.20-2.95$ \\
\hline Lactation 4+ & 3.34 & 0.250 & 16.25 & 0.000 & $2.89-3.87$ \\
\hline
\end{tabular}

model with several predictor variables, including BVD, Nocardia, 6 lactation groups, and region of Canada. It is possible that the association between BLV and longevity may have been diminished by inclusion of additional diseases that may have at least partially been associated with BLV.

In another Canadian study (Jacobs et al., 1995), the cull rate among BLV-positive cattle was $27 \%$ greater than among BLV-negative cattle, although this was not statistically significant. The cull rate among older cattle was higher among BLV-positive cattle than among BLV negatives. However, this effect was counteracted in their combined analysis by a reverse association among cattle $<3.5$ yr of age in which the culling rate among the BLV-positive cattle was substantially lower than the rate in the BLV-negative cattle. This finding is in agreement with our results, in that we also found no significant effect of BLV when our analysis was limited to only first-lactation animals.

Brenner et al. (1989) found that BLV infection was associated with reduced milk production, reduced reproductive efficiency, and a cull rate among BLVpositive cattle that was 3.6 times that of the seronegative cattle. Trainin et al. (1996) reported a cull rate among BLV-positive cattle that was 3.61 times the rate in BLV-negative cattle. Pollari et al. (1993) reported that, among older cows, BLV-infected cows were culled before their uninfected herd mates. In a study of a single herd, Rhodes et al. (2003) found that BLV seroprevalence was not associated with cow survival.

Da et al. (1993) reported results suggesting that genetic resistance to persistent lymphocytosis was associated with cow longevity. A major histocompatibility complex class I (BoLA-A) allele that is associated with resistance to persistent lymphocytosis (PL) is also associated with increased milk production potential and longevity (Da et al., 1993). Wu et al. (1989) reported that BLV-infected cows with high genetic potentials for milk and fat yields were more susceptible to becoming PL than were cows with lower genetic potential, and PL cows do not produce milk or fat according to predicted genetic values. If genes fostering a susceptibility to BLV immunosuppression have been increasing in the US dairy population, it is possible that the impact of BLV is now greater than it was in previous years.

Analysis of the effects of BLV on cow longevity is complicated by confounding with age (lactation number or parity). Older cows are more likely to be culled

Table 2. Survival of cattle following bovine leukemia virus (BLV) testing for milk ELISA antibodies

\begin{tabular}{|c|c|c|c|c|c|}
\hline Effect & $\begin{array}{c}\text { Hazard } \\
\text { ratio }\end{array}$ & SE & $\mathrm{Z}^{1}$ & $P$-value & $95 \%$ CI \\
\hline $\mathrm{OD}<0.1^{2}$ & - & - & 一 & - & - \\
\hline $0.1 \leq \mathrm{OD}<0.25^{3}$ & 1.08 & 0.832 & 1.01 & 0.314 & $0.929-1.26$ \\
\hline $0.25 \leq \mathrm{OD}<0.5^{4}$ & 1.25 & 0.0857 & 3.21 & 0.001 & $1.09-1.43$ \\
\hline $\mathrm{OD}>0.50^{5}$ & 1.40 & 0.107 & 4.36 & 0.000 & $1.20-1.63$ \\
\hline Lactation 1 & - & - & - & - & - \\
\hline Lactation 2 & 1.72 & 0.135 & 6.94 & 0.000 & $1.48-2.01$ \\
\hline Lactation 3 & 2.54 & 0.193 & 12.3 & 0.000 & $2.19-2.95$ \\
\hline Lactation $4+$ & 3.32 & 0.249 & 16.03 & 0.000 & $2.87-3.85$ \\
\hline
\end{tabular}




\section{Product-Limit Survival Estimates}

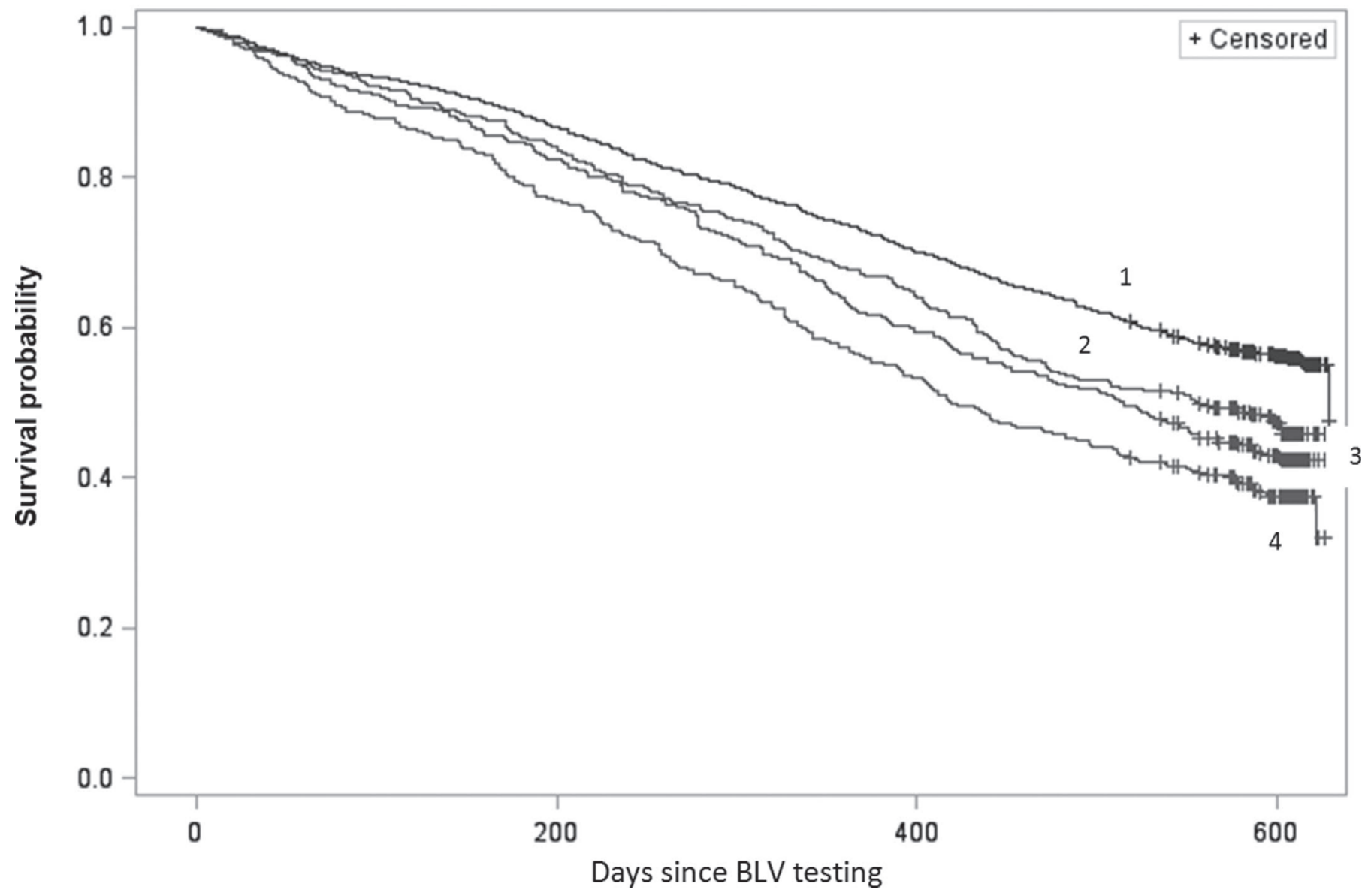

Figure 2. Survival of cattle following bovine leukemia virus (BLV) testing; proportion of cattle surviving (y-axis) and days since BLV testing (x-axis). ${ }^{1} \mathrm{BLV}$ negative (optical density $\left.<0.1\right) ;{ }^{2} \mathrm{BLV}$ positive $(0.1 \leq$ optical density $<0.25) ;{ }^{3} \mathrm{BLV}$ positive $(0.25 \leq$ optical density $<0.5)$; ${ }^{4} \mathrm{BLV}$ positive (optical density $\geq 0.5$ ).

and are also more likely to have antibodies for BLV (Erskine et al., 2012c), yet we showed that herds with higher rates of BLV tend to contain younger cows (Erskine et al., 2012a). As such, cows with BLV are more likely to be older, but herds with high BLV prevalence are more likely to be populated with younger cows due to poor cow longevity. Therefore, studies of the association between BLV and cow longevity must either be stratified by cow age (or parity) or be adjusted in some other way for the confounding effects of age.

Herd effects and population dynamics are also of major importance in affecting cow longevity. We previously reported for this same database that the herdlevel cow removal rate (combination of died and culled cows) was not associated with BLV prevalence; however, herds with higher rates of BLV had significantly younger cattle (Erskine et al., 2012a). Depending on the study design, cull rate may not be a good measure of cow longevity. Two herds maintaining constant herd size and experiencing the same cull rate may have both reached an equilibrium; however, the first herd may have reached this steady state with a population of older cattle and may enjoy the opportunity for more discretionary culling, whereas the second herd may have younger cattle and be constantly struggling to maintain herd size due to more nondiscretionary culls. It is, therefore, important that herd effects be included in the analysis of cow-level point-prevalence studies so that the effect of BLV infection is made within herd (i.e., among herd mates).

Additionally, the prevailing theory supports that recently infected animals probably show little deleterious effect from BLV. Lymphocytosis is the standard predictor of disease progression, although it is not necessarily associated with pathogenic change (Rodriguez, et al., 2011). However, several reports have suggested the use of PCR or proviral load as alternative indicators for disease progression (Fechner et al., 1997; Rodríguez et 
al., 2009; Jimba et al., 2010). When lymphocyte count is not available, the lactation number of infected cattle may provide a crude proxy in that older animals are more likely to have infections of longer duration, and, therefore, their BLV infections are more likely to have progressed to the point of PL. Independent of cow age, BLV ELISA OD results may also indicate a progression of BLV pathogenesis toward immunosuppression, as demonstrated by a dose-dependent increased risk of being culled.

Although the interaction between lactation and BLV positivity was not significant, BLV-positive first-lactation cows, when analyzed by themselves, did not have significantly reduced longevity compared with their negative herd mates. It appears that BLV-associated effects on survival are more evident in later lactations when BLV-positive cows are more likely to have infections of longer duration.

Our finding that BLV was highly associated with reduced cow longevity supports the conclusion, already reached by many other nations, that BLV control or eradication might improve dairy production. Considerable expense is incurred in raising a dairy cow to approximately 2 yr of age when they start producing milk. Herds with poor longevity quickly find themselves populated primarily with first- and second-lactation animals, which have not yet achieved their full milk production potential (Erskine et al., 2012a).

In addition to the impact on farm profitability, cow longevity is an important indicator of animal welfare just as the average years of life expectancy is a common measure of human well-being. The ideal life for a dairy animal is one that is long, comfortable, healthy and free of pain until she is humanely stunned and slaughtered. If severely immunosuppressed, it is likely that BLV-infected cattle suffer considerably during the last months of their lives. Further research is needed to fully describe the health status of BLV-infected cattle in the months before they are culled from their herds.

The association between BLV and reduced longevity showed a marked dose response, which supports the theory that this association is causal. Diagnostic laboratories and other testing facilities may wish to provide OD values as well as a simple positive or negative results, as higher OD values were found to be significantly more predictive of reduced longevity than were lower OD values. However, no evidence exists to suggest that high OD values should be used to direct culling decisions, as high-OD cattle may not necessarily be more infectious than infected cattle with lower OD values. Instead, evidence exists that management practices, of the type used to eradicate this disease in other countries, may be effective in reducing the impact of BLV on our dairy industry (Erskine et al., 2012b).

\section{CONCLUSIONS}

Cattle positive for milk ELISA BLV antibodies were found to be at significantly higher risk of leaving their herds in the approximately $1.6 \mathrm{yr}$ since BLV testing. A dose response was demonstrated among BLV-positive cattle, in that cattle with higher ELISA OD values were at greater risk of leaving the herd than were cattle with lower OD values. Control of BLV in the United States may be deemed cost effective when future studies estimate the total economic impact of BLV by considering losses to exports, condemnations, milk production, reproductive efficiency, and cow longevity.

\section{ACKNOWLEDGMENTS}

The authors gratefully acknowledge funding of this project by the Elwood Kirkpatrick Dairy Science Research Endowment (Michigan State University, East Lansing) and technical support by Antel BioSystems Inc. (Lansing, MI).

\section{REFERENCES}

Acaite, J., V. Tamosiunas, K. Lukauskas, J. Milius, and J. Pieskus. 2007. The eradication experience of enzootic bovine leukosis from Lithuania. Prev. Vet. Med. 82:83-89.

Brenner, J., M. Van-Haam, D. Savir, and Z. Trainin. 1989. The implication of BLV infection in the productivity, reproductive capacity and survival rate of a dairy cow. Vet. Immunol. Immunopathol. $22: 299-305$.

Cleves, M., R. G. Gutierrez, W. Gould, and Y. Marchenko. 2010. An Introduction to Survival Analysis Using Stata. 3rd ed. Stata Press, College Station, TX.

Da, Y., R. D. Shanks, J. A. Stewart, and H. A. Lewin. 1993. Milk and fat yields decline in bovine leukemia virus-infected Holstein cattle with persistent lymphocytosis. Proc. Natl. Acad. Sci. USA 90:6538-6541.

Erskine, R. J., P. C. Bartlett, T. M. Byrem, C. L. Render, C. Febvay, and J. T. Houseman. 2012a. Association between bovine leukemia virus, production, and population age in Michigan dairy herds. J. Dairy Sci. 95:727-734.

Erskine, R. J., P. C. Bartlett, T. M. Byrem, C. L. Render, C. Febvay, and J. T. Houseman. 2012b. Herd-level determinants of bovine leukaemia virus prevalence in dairy farms. J. Dairy Res. 79:445-450.

Erskine, R. J., P. C. Bartlett, T. M. Byrem, C. L. Render, C. Febvay, and J. T. Houseman. 2012c. Using a herd profile to determine age-specific prevalence of bovine leukemia virus in Michigan dairy herds. Vet. Med. Int. 2012:350374.

European Commission. 2003. European Commission Decision 2003/467/EC establishing the official tuberculosis, brucellosis, and enzootic-bovine-leukosis-free status of certain Member States and regions of Member States as regards bovine herds (notified under document number C(2003) 1925). Accessed July 4, 2012. http:// faolex.fao.org/docs/pdf/eur38376.pdf.

Fechner, H., P. Blankenstein, A. C. Looman, J. Elwert, L. Geue, C. Albrecht, A. Kurg, D. Beier, O. Marquardt, and D. Ebner. 1997. Provirus variants of the bovine leukemia virus and their relation to the serological status of naturally infected cattle. Virology 237:261-269.

Huber, N. L., R. F. DiGiacomo, J. F. Evermann, and E. Studer. 1981. Bovine leukemia virus infection in a large Holstein herd: prospective comparison of production and reproductive performance in antibody-negative and antibody-positive cows. Am. J. Vet. Res. 42:1477-1481. 
Jacobs, R. M., F. L. Pollari, W. B. McNab, and B. Jefferson. 1995. A serological survey of bovine syncytial virus in Ontario: Associations with bovine leukemia and immunodeficiency-like viruses, production records, and management practices. Can. J. Vet. Res. $59: 271-278$

Jimba, M., S. Takeshima, K. Matoba, D. Endoh, and Y. Aida. 2010. BLV-CoCoMo-qPCR: Quantitation of bovine leukemia virus proviral load using the CoCoMo algorithm. Retrovirology 7:91.

Nuotio, L., H. Rusanen, L. Sihvonen, and E. Neuvonen. 2003. Eradication of enzootic bovine leukosis from Finland. Prev. Vet. Med. 59:43-49.

Ott, S. L., R. Johnson, and S. J. Wells. 2003. Association between bovine-leukosis virus seroprevalence and herd-level productivity on US dairy farms. Prev. Vet. Med. 61:249-262.

Pollari, F. L., R. F. DiGiacomo, and J. F. Evermann. 1993. Use of survival analysis to compare cull rates between bovine leukemia virus seropositive and seronegative dairy cows. Am. J. Vet. Res. 54:1400-1403.

Pollari, F. L., V. L. Wangsuphachart, R. F. DiGiacomo, and J. F. Evermann. 1992. Effects of bovine leukemia virus infection on production and reproduction in dairy cattle. Can. J. Vet. Res. $56: 289-295$

Rhodes, J.K., K. D. Pelzer, Y. J. Johnson, and E. Russek-Cohen. 2003. Comparison of culling rates among dairy cows grouped on the basis of serologic status for bovine leukemia virus. J. Am. Vet. Med. Assoc. 223:229-231.

Rodríguez, S. M., A. Florins, N. Gillet, A. de Brogniez, M. T. SánchezAlcaraz, M. Boxus, F. Boulanger, G. Gutiérrez, K. Trono, I. Alvarez, L. Vagnoni, and L. Willems. 2011. Preventive and therapeutic strategies for bovine leukemia virus: Lessons for HTLV. Viruses 3:1210-1248

Rodriguez, S. M., M. D. Golemba, R. H. Campos, K. Trono, and L. R. Jones. 2009. Bovine leukemia virus can be classified into seven genotypes: Evidence for the existence of novel clades. J. Gen. Virol. 90:2788-2797.

Sargeant, J. M., D. F. Kelton, S. W. Martin, and E. D. Mann. 1997. Associations between farm management practices, productivity, and bovine leukemia virus infection in Ontario dairy herds. Prev. Vet. Med. 31:211-221.

Schwartz, I., and D. Levy. 1994. Pathobiology of bovine leukemia virus. Vet. Res. 25:521-536.

Thurmond, M. C., C. B. Maden, and R. L. Carter. 1985. Cull rates of dairy cattle with antibodies to bovine leukemia virus. Cancer Res. 45:1987-1989.

Tiwari, A., J. A. VanLeeuwen, I. R. Dohoo, H. Stryhn, G. P. Keefe, and J. P. Haddad. 2005. Effects of seropositivity for bovine leukemia virus, bovine viral diarrhoea virus, Mycobacterium avium subspecies paratuberculosis, and Neospora caninum on culling in dairy cattle in four Canadian provinces. Vet. Microbiol. 109:147-158.

Trainin, Z., J. Brenner, R. Meirom, and H. Ungar-Waron. 1996. Detrimental effect of bovine leukemia virus (BLV) on the immunological state of cattle. Vet. Immunol. Immunopathol. 54:293-302.

Trono, K. G., D. M. Pérez-Filgueira, S. Duffy, M. V. Borca, and C. Carrillo. 2001. Seroprevalence of bovine leukemia virus in dairy cattle in Argentina: Comparison of sensitivity and specificity of different detection methods. Vet. Microbiol. 83:235-248.

VanLeeuwen, J. A., L. Forsythe, A. T. Tiwari, and R. Chartier. 2005 Seroprevalence of antibodies against bovine leukemia virus, bovine viral diarrhea virus, Mycobacterium avium subspecies paratuberculosis, and Neospora caninum in dairy cattle in Saskatchewan. Can. Vet. J. $46: 56-58$

Wu, M. C., R. D. Shanks, and H. A. Lewin. 1989. Milk and fat production in dairy cattle influenced by advanced subclinical bovine leukemia virus infection. Proc. Natl. Acad. Sci. USA 86:993-996. 